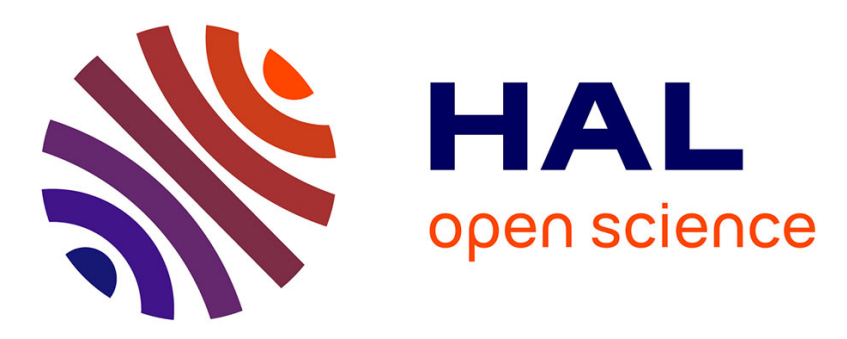

\title{
3D integrated geophysical modeling for the 2008 magma intrusion at Etna: constraints on rheology and dike overpressure
}

Gilda Currenti, Rosalba Napoli, Agnese Di Stefano, Filippo Greco, Ciro del Negro

\section{To cite this version:}

Gilda Currenti, Rosalba Napoli, Agnese Di Stefano, Filippo Greco, Ciro del Negro. 3D integrated geophysical modeling for the 2008 magma intrusion at Etna: constraints on rheology and dike overpressure. Physics of the Earth and Planetary Interiors, 2011, 185 (1-2), pp.44. 10.1016/j.pepi.2011.01.002 . hal-00727169

\section{HAL Id: hal-00727169 \\ https://hal.science/hal-00727169}

Submitted on 3 Sep 2012

HAL is a multi-disciplinary open access archive for the deposit and dissemination of scientific research documents, whether they are published or not. The documents may come from teaching and research institutions in France or abroad, or from public or private research centers.
L'archive ouverte pluridisciplinaire HAL, est destinée au dépôt et à la diffusion de documents scientifiques de niveau recherche, publiés ou non, émanant des établissements d'enseignement et de recherche français ou étrangers, des laboratoires publics ou privés. 


\section{Accepted Manuscript}

Title: 3D integrated geophysical modeling for the 2008

magma intrusion at Etna: constraints on rheology and dike

overpressure

Authors: Gilda Currenti, Rosalba Napoli, Agnese Di Stefano, Filippo Greco, Ciro Del Negro

PII:

S0031-9201(11)00012-4

DOI: doi:10.1016/j.pepi.2011.01.002

Reference: PEPI 5370

To appear in: Physics of the Earth and Planetary Interiors

Received date:

26-5-2010

Revised date:

Accepted date:

$12-1-2011$

Please cite this article as: Currenti, G., Napoli, R., Di Stefano, A., Greco, F., Del Negro, C., 3D integrated geophysical modeling for the 2008 magma intrusion at Etna: constraints on rheology and dike overpressure, Physics of the Earth and Planetary Interiors (2010), doi:10.1016/j.pepi.2011.01.002

This is a PDF file of an unedited manuscript that has been accepted for publication. As a service to our customers we are providing this early version of the manuscript. The manuscript will undergo copyediting, typesetting, and review of the resulting proof before it is published in its final form. Please note that during the production process errors may be discovered which could affect the content, and all legal disclaimers that apply to the journal pertain. 


\title{
3D integrated geophysical modeling for the 2008 magma intrusion at
}

\section{Etna: constraints on rheology and dike overpressure}

\author{
Gilda Currenti ${ }^{1}$, Rosalba Napoli ${ }^{1}$, Agnese Di Stefano ${ }^{1,2}$, Filippo Greco ${ }^{1}$, Ciro Del Negro ${ }^{1}$ \\ (1) Istituto Nazionale di Geofisica e Vulcanologia - Sezione Catania, Italy \\ (2) Dipartimento di Ingegneria Elettrica, Elettronica e dei Sistemi, Università di Catania, Italy
}

\begin{abstract}
We present a 3D numerical model based on FEM (Finite Element Method) to jointly evaluate geophysical changes caused by dislocation and overpressure sources in volcanic areas. A coupled numerical problem was solved to estimate ground deformation, gravity and magnetic changes produced by stress redistribution accompanying magma migration within the volcano edifice. We successfully applied the integrated numerical procedure to image the magmatic intrusion occurring in the northern flank of Etna during the onset of the 2008 eruption. A multi-layered crustal structure of the volcano constrained by geological models and geophysical data was considered. Geodetic and gravity data provide information on the strain field, while piezomagnetic changes give constraints on the stress field. Therefore, the integrated modeling gives insights on Mt Etna rheology and dike overpressure involved in the magma propagation and improves understanding of dike emplacement in the northern sector of the volcano. Our FEM-based approach improves the reliability of model-based inference of geophysical parameters obtained during monitoring of the onset of Etna lateral intrusions that can prelude to an impending eruption.
\end{abstract}

Key words: Numerical method, geophysical modeling, Etna volcano, magmatic intrusion.

\section{Introduction}

Magma migration inside a volcano edifice generates a wide variety of geophysical signals, which can be observed before and during eruptive processes. In particular, ground deformation, gravity and magnetic changes in volcanic areas are generally recognized as reliable indicators of unrest, resulting from the intrusion of fresh magma within the shallow rock layers. Continuous measurements of these geophysical signals are useful for imaging the spatio-temporal evolution of propagating dikes, and revealing quantitative information about the dike geometry and its overpressure (Segall et al., 2001; Furuya et al., 2003; Ebinger et al., 2010; Del Negro et al., 2004). These geophysical signals are generally interpreted separately from each other and the consistency of interpretations from these different methods is qualitatively checked only a posteriori. An integrated approach based on different geophysical data should prove a more 
efficient and accurate procedure for inferring magmatic intrusions and minimizing interpretation ambiguities.

Volcano monitoring at Mt Etna has reached a high quality level, and advanced monitoring networks enabled collecting multi-disciplinary data during the frequent eruptions in recent years. Particularly, geodetic, gravity and magnetic investigations have played an increasingly important role in studying the eruptive processes (Napoli et al., 2008; Bonforte et al., 2008; Carbone et al., 2007; Del Negro et al., 2004). The comparison between these observations and geophysical models allowed inferences about volcanic source parameters and detailed descriptions of magma migration, but limited efforts have been made for effective integration of these different data. Ground deformation, gravity and magnetic variations due to volcanic sources have been modeled separately using analytical solutions, based on simple homogeneous elastic half-space models (Bonaccorso and Davis, 2004; Carbone et al 2007; Del Negro and Currenti, 2003; Del Negro et al 2004). However, as indicated by geological evidences and seismic tomography (Chiarabba et al., 2000; Tibaldi and Groppelli, 2002), Etna volcano is elastically inhomogeneous and rigidity layering and heterogeneities are likely to affect the magnitude and pattern of observed signals (Currenti et al., 2007; 2009).

To overcome these intrinsic limitations and provide more realistic models, which allow considering topographic effects as well as heterogeneous distribution of medium properties, we developed a numerical procedure based on Finite Element Method (FEM). This procedure allows joint evaluation of geophysical changes caused by dislocation and overpressure sources in a 3D formulation. The 2008-2009 Etna eruption offers an exemplary case study to validate the capability of the proposed integrated approach for imaging the intrusive process occurring in the northern flank of the volcano (Napoli et al., 2008; Aloisi et al., 2009). We review the previous analytical model obtained by Napoli et al. (2008) by constructing a 3D numerical model to consider the magneto-elastic heterogeneities and the real topography of the volcano. Numerical solutions for deformation, gravity and magnetic fields are obtained by modeling the source intrusion as an extension fracture driven by a magmatic overpressure, which is a realistic representation of an intrusive dike. Combined deformation, gravity, and magnetic investigations provide insights on Mt Etna rheology and dike overpressure involved during magma propagation, which are found to be within a range of values in agreement with rock strength data measured in the laboratory.

\section{The 2008-2009 Etna eruption}

The onset of the 2008-2009 eruptive activity was accompanied by a superficial seismic swarm taking place in a NNW-SSE elongated area at the eastern base of Mt Etna summit craters. On $13^{\text {th }}$ May 2008, epicenters time patterns evidenced an almost stationary distribution in the upper flanks 
of the Valle del Bove until 9:30 GMT, then a clear migration of the seismic events occurred toward the top of the North-East Rift, suggesting a northward propagation of a magmatic intrusion. Earthquakes were accompanied by a gradual and intense increase in the intensity of volcanic tremor (Di Grazia et al., 2009). The volcanic activity was characterized by the opening of a fracture field on the northern flank of the volcano (Fig. 1), and an E-W eruptive fissure in the upper sides of the Valle del Bove (Napoli et al., 2008; Aloisi et al., 2009). Remarkable changes in the local magnetic field were observed in coincidence with the seismic swarm onset. No significant variations were observed before $13^{\text {th }}$ May, while large negative changes in local magnetic field (the amplitude ranges between -1.8 and $-6.5 \mathrm{nT}$ ) occurred at the stations placed on the northern flank of the volcano within a few hours coinciding with the quick epicentral migration of the seismic events from Valle del Bove toward the North-East Rift (Napoli et al., 2008). Discrete gravity measurements were carried out at 19 benchmarks along a profile that lies between 500 and 2200 $\mathrm{m}$ a.s.I. of altitude on the north-eastern flank of the volcano. Measurements gathered on $14^{\text {th }}$ May 2008 were referenced to the gravity survey performed on $5^{\text {th }}$ May, but no significant variations were observed (Budetta et al., 2008).

Remarkable ground deformation was recorded by permanent tilt and GPS networks. Changes started first in the summit area where the largest variations (about $100 \mu \mathrm{rad}$ ) were detected, and successively were observed in the middle flanks of the volcano but with much smaller amplitudes. The summit stations of the continuously recording GPS network detected horizontal displacements of a few tens of centimetres. Smaller variations of a few centimetres were detected at the other stations. The deformation pattern was radial with respect to the summit area (Aloisi et al., 2009).

Preliminary analytical solutions were used to model the geophysical observations and infer the intrusion source parameters. Magnetic data inversion indicated the response to a tensile mechanism with an intrusion crossing the volcano edifice along a ca. NNW-SSE direction in the northern flank attributable to piezomagnetic effects. The estimated intrusive dike, located within the zone of the seismic swarm that occurred during magma propagation, engenders a deformation pattern that well fits also the ground deformation recorded by the continuous GPS network operating on Mt Etna. Gravity changes expected from the same intrusive dyke are below data uncertainty at the gravity benchmarks confirming a lack of significant variations in the investigated area (Napoli et al., 2008). Also geodetic data inversion evidenced a shallow intrusion propagating laterally to the NNW direction from the central conduit (Aloisi et al., 2009). In contrast, the E-W eruptive fissure in the Valle del Bove could have given only minor contribution to stress and strain changes probably because of the highly fractured and compliant rocks of this area. Indeed, magma intruded toward the Valle del Bove without any seismic events and lava effused almost passively (Napoli et al., 2008; Aloisi et al., 2009).

Even if the analytical models provided an acceptable representation of the magma intrusion, they do not explain the observed geophysical variations in details because a simple elastic half-space 
assumption was used. Therefore, we developed a 3D numerical procedure based on FEM including the real topography of the Etna and the magneto-elastic heterogeneity to jointly evaluate ground deformation, magnetic and gravity changes caused by the magma intrusion.

\section{Ground deformation, magnetic and gravity solutions}

Magma ascent to the Earth's surface forces surrounding crustal rocks apart and this perturbs stress and displacement fields, commonly producing variations in the magnetization of rocks and modifications in the density distribution.

The variations in the magnetization of rocks induced by stress redistribution produce a local magnetic field of about few nanoteslas, known as piezomagnetic field (Nagata, 1970; Pozzi, 1977). Piezomagnetic changes are calculated by combining the Cauchy-Navier equation for elastic equilibrium and the Poisson's equation for the magnetic potential (Sasai 1986; Sasai, 1991; Utsugi et al. 2000). The change $\boldsymbol{\Delta} \boldsymbol{J}$ in rock magnetization $\boldsymbol{J}$ at an arbitrary point, associated with mechanical stress $\sigma$, can be expressed as follows:

$$
\begin{aligned}
& \Delta \mathbf{J}=\frac{3}{2} \beta \mathbf{T} \cdot \mathbf{J} \\
& \mathbf{T}_{k l}=\sigma_{k l}-\frac{1}{3} \delta_{k l} \Theta \\
& \Theta=\sigma_{x x}+\sigma_{y y}+\sigma_{z z}
\end{aligned}
$$

where $\beta$ is the stress sensitivity, $\sigma_{k l}$ are the components of the stress tensor, $\delta_{k l}$ is the Kronecker delta. The piezomagnetic change depends on the deviatoric stress tensor $\boldsymbol{T}$, that is obtained from the stress tensor $\boldsymbol{\sigma}$ subtracting the isotropic stress $\frac{1}{3} \delta_{k l} \Theta$. Using Hooke's law, the components of stress tensor can be expressed in terms of the displacement vector as:

$$
\sigma_{k l}=\lambda \delta_{k l} \nabla \cdot \mathbf{u}+\mu\left(\frac{\partial u_{k}}{\partial x_{l}}+\frac{\partial u_{l}}{\partial x_{k}}\right)
$$

where $\lambda$ and $\mu$ are the Lamé's constants and $\boldsymbol{u}$ is the displacement field. Substituting Eq. (2) in Eq. (1) we obtain the stress induced magnetization expressed by displacement components $\left(\Delta J_{k l}\right.$ is the l-th component of the incremental magnetization produced by the $k$-th component of the initial magnetization):

$$
\Delta J_{k l}=J_{k} \beta \mu\left[\frac{3}{2}\left(\frac{\partial u_{k}}{\partial x_{l}}+\frac{\partial u_{l}}{\partial x_{k}}\right)-\delta_{k l} \nabla \cdot \mathbf{u}\right]
$$


For magnetostatic problems, where no currents are present, the problem can be solved using a scalar piezomagnetic potential $W_{k}$, related to piezomagnetic field $\boldsymbol{H}$, which satisfies the magnetostatic Poisson equation:

$$
\nabla^{2} W_{k}=-\nabla \cdot \mathbf{H}=\nabla \cdot \Delta \mathbf{J}_{\mathbf{k}}
$$

Magma migration is also accompanied by density redistribution in the medium that can engender significant variations in the gravity field ranging in amplitude between a few $\mu \mathrm{Gal}$ and several hundred $\mu \mathrm{Gal}$ with a spectrum varying from 1 second to more than 1 year (Hinderer and Crossley, 2000; Greco et al., 2008). The gravity change $\delta g$, related to the density redistribution, can be calculated by solving the following Poisson's differential equation for the gravitational potential $\phi_{\mathrm{g}}$ (Cai \& Wang 2005):

$$
\nabla^{2} \phi_{g}=-4 \pi G \Delta \rho(x, y, z)
$$

where $G$ denotes the universal gravitational constant and $\Delta \rho(x, y, z)$ is the change in the density distribution. Generally, the total gravity change at a benchmark on the ground surface is given by:

$$
\delta g(x, y, z)=-\frac{\partial \phi_{g}}{\partial z}+\delta g_{0}
$$

where $\delta g_{0}$ represents the "free air" gravity change accompanying the uplift of the observation site. The "free air" gravity change, in first approximation, is given by:

$$
\delta g_{0}=-\gamma \delta h
$$

where $\gamma$ is the free-air gravity gradient (generally $\gamma=308.6 \mu \mathrm{Gal} / \mathrm{m}$ ) and $\delta h$ the elevation change. The density variations related to the subsurface mass redistribution can be accounted for by three main terms:

$$
\Delta \rho(x, y, z)=\delta \rho_{1}-\mathbf{u} \cdot \nabla \rho_{0}-\rho_{0} \nabla \cdot \mathbf{u}
$$

where $\rho_{0}$ is the embedding medium density and $\delta \rho_{1}$ is the density change due to the input of intrusive mass from remote distance. The first term originates from the density change related to the introduction of the new mass into the displaced volume. The second term is due to the displacement of density boundaries in heterogeneous media, and the third term is the contribution due to the volume change arising from compressibility of the surrounding medium (Bonafede \& Mazzanti 1998). Each term in the density variation contributes to the total gravity change observed at the ground surface. Therefore, the gravity changes are made up by four different contributions:

$$
\delta g=\delta g_{0}+\delta g_{1}+\delta g_{2}+\delta g_{3}
$$

where $\delta g_{1}, \delta g_{2}$ and $\delta g_{3}$ arise from the three terms in Eq. 8. The $\delta g_{2}$ and $\delta g_{3}$ terms highlight that the computation of the displacement field at depth is required in order to evaluate these gravity contributions. This means that changes in the gravity field cannot be interpreted only in terms of 
additional mass input, disregarding the deformations of the surrounding rocks (Bonafede and Mazzanti 1998; Currenti et al., 2007; Charco et al., 2009). Since gravity and piezomagnetic changes are strictly related to stress and deformation fields, ground deformation, magnetic and gravity changes produced by volcanic sources need to be jointly modeled (Sasai, 1991; Okubo and Oshiman 2004; Hagiwara, 1977).

We developed a coupled numerical model using the Finite Element Method (FEM) to compute the deformation, gravity and magnetic changes caused by dislocation and pressure sources. The FEM is suitable to easily solve the model equations and to account for topography and medium heterogeneity, which can alter the estimate of the investigated geophysical changes. We solve the model equations in three steps. Using the commercial software COMSOL Multiphysics (2008) we numerically solve: (i) the elastostatic problem for the elastic deformation field and its derivatives, (ii) the coupled Poisson's problem for gravity field (Currenti et al., 2007), (iii) the coupled Poisson's problem for piezomagnetic potential field (Currenti et al., 2009).

\section{Numerical model of the 2008 magmatic intrusion at Etna}

The numerical procedure was applied to model the 2008 magmatic intrusion occurring along the north flank of Mt Etna during the initial phase of the eruption. A fully three-dimensional elastic finite element model of Mt Etna was designed to evaluate the ground deformation, magnetic and gravity changes.

\subsection{Numerical model of Etna}

A computational domain with a length, breadth and width of $100 \times 100 \times 50 \mathrm{~km}^{3}$ is considered for the deformation field calculations. The 3D topography of Mt Etna, which is rather asymmetric with a prominent mass deficit in correspondence of Valle del Bove, was taken into account using a digital elevation model from the $90 \mathrm{~m}$ Shuttle Radar Topography Mission (SRTM) data and a bathymetry model from the GEBCO database (http://www.gebco.net/). The computational domain was meshed into 215,009 isoparametric, and arbitrarily distorted tetrahedral elements connected by 38,007 nodes. Lagrange cubic shape functions are used in the computations, since the use of lower order elements worsens the accuracy of stress field solutions. Zero displacements are assigned at the bottom and the lateral boundaries of the domain, while the upper boundary representing the ground surface is stress free. The intrusion source is simulated as a discontinuity surface by introducing the mesh nodes in pairs along the surface rupture.

In order to solve the Poisson's equations (Eqs. 4 and 5), the potential or its normal derivatives are assigned at the boundaries of the domain (Dirichlet or Neumann boundary conditions, respectively), which is extended along the $z$ direction to $50 \mathrm{~km}$ to finally obtain a $100 \times 100 \times 100 \mathrm{~km}^{3}$ 
computational domain for guaranteeing continuity of the gravity and magnetic potential on the ground surface. Along the external boundaries, zero gravity potential is specified using Dirichlet boundary conditions, while the magnetic field is assumed to be tangential by assigning a Neumann condition on the magnetic potential. The magnetic problem is made unique by setting the potential to zero at an arbitrary point on the external boundary.

Heterogeneous distribution of magneto-elastic properties is included in the model. Considering the tomographic image of Etna plumbing system obtained by Chiarabba et al. (2000) and the geological models by Tibaldi and Groppelli (2002), we evaluate a three-layered half-space structure. For the first layer, that extends from ground surface to $1 \mathrm{~km}$ asl, we assume a value of Young modulus of $17 \mathrm{GPa}$, reproducing the low rigidity of the rocks in the volcano edifice. For the second layer, from $1 \mathrm{~km}$ asl to $4 \mathrm{~km}$ bsl, we assume a value of $40 \mathrm{GPa}$ that represents the rigidity of a sublayer with different properties. Finally, the third layer, which extends from $4 \mathrm{~km}$ bsl to the bottom of the domain, is supposed with a higher rigidity with value of $80 \mathrm{GPa}$. The Poisson's ratio is assumed homogeneous in the entire domain and has a value of 0.25 . We do not include heterogeneity in the rock density properties since density models available at Etna have low resolution and accuracy (Corsaro and Pompilio, 2004; Schiavone and Loddo, 2007), especially at shallow depth where the intrusion occurred. To estimate the gravity changes, we hypothesize a medium with homogeneous density $\rho_{\mathrm{c}}=2500 \mathrm{~kg} / \mathrm{m}^{3}$. Heterogeneous distribution of initial magnetization is also used in the piezomagnetic model. We assume a value of the initial magnetization free from stress $J_{0}=8 \mathrm{~A} / \mathrm{m}$ from ground surface to $1 \mathrm{~km}$ asl, reproducing the magnetization of the rocks in the volcano edifice (Currenti et al., 2009). The second layer starts with a initial magnetization value of $J_{0}=5 \mathrm{~A} / \mathrm{m}$ that decreases in depth with the geothermal gradient until reaching the depth of the Curie isotherm $\left(T_{C} \approx 550^{\circ} \mathrm{C}\right.$ for Etna's rocks) at about $18 \mathrm{~km}$ (Currenti et al., 2009).

\subsection{Numerical results}

The preliminary numerical solution (model A) was obtained using the source geometry obtained by the analytical inversion model by Napoli et al. (2008). In this way we can appreciate how the heterogeneous distribution of magneto-elastic parameters in the medium and the topography make the numerical results differ from the simple analytical solutions from a homogeneous half-space model having an elastic rigidity $\mu=30 \mathrm{GPa}$, Poisson ratio $v=0.25$ and density $\rho=2500 \mathrm{~kg} / \mathrm{m}^{3}$. It is worth noting that we do not invert either the geodetic data or the magnetic and gravity data, but we assume the intrusion source to estimate the expected geophysical changes. A dislocation model was simulated assigning an opening of $2 \mathrm{~m}$ along the discontinuity surface striking $\mathrm{N} 20^{\circ} \mathrm{W}$, dipping $80^{\circ} \mathrm{E}$, with a length of $2.5 \mathrm{~km}$, width $2 \mathrm{~km}$, representing the magma intrusion.

Numerical results compared to analytical solutions reveal slight differences in pattern and intensity of the deformation field at the summit GPS stations (Figs. 2a and 2b). However, stations located at 
lower altitude show a higher misfit with respect to the half-space homogeneous model. At mid-flank stations, where the role of topography is clearly very important, the horizontal deformation decreases and the match with the observations improves. In contrast, the piezomagnetic field changes show significant deviations from the homogeneous half-space solution. Significant discrepancies are observed at most of the magnetic stations (Fig. 3). The symmetry of total magnetic intensity observed in the analytical model is lost in the numerical result (Fig. 2b). The shape of the piezomagnetic field is strongly modified in the eastern sector of the volcano in correspondence with the rugged topography. The piezomagnetic field is wider and more intense than the analytical one in the northern flank, and significant discrepancies, up to $5 \mathrm{nT}$, between the numerical and analytical models are observed at the magnetic stations (Fig. 3). In particular, the highest discrepancies are observed at BVD and PDN stations. In the numerical model the amplitude of the piezomagnetic field increases at BVD and PDN stations, while it decreases at $B C N$. Therefore, a higher misfit with respect to the magnetic observations is obtained in the numerical model A. Each contribution to the gravity field (see Eq. 9) was investigated separately using both the analytical (Okubo, 1992) and numerical solutions to understand their own effects (Figs. 4 and 5). Numerical solutions (Figs. 5), as well as the analytical ones (Figs. 4), show significant variations only in proximity of the magmatic intrusion, where, unfortunately, no data are available. The predominant contribution is the $\delta g_{2}$ term, originating from the displacement of density boundaries at the ground surface (Figs. $4 b$ and $5 b$ ). Even if a magmatic source with a density contrast of $\Delta \rho=200 \mathrm{~kg} / \mathrm{m}^{3}$ is assumed, the $\delta g_{1}$ contribution (Figs. 4 a and $5 \mathrm{a}$ ) related to the mass intrusion attains an amplitude less than $10 \mu \mathrm{Gal}$. This contribution is comparable with $\delta g_{3}$ (Figs. $4 \mathrm{c}$ and $5 \mathrm{c}$ ) produced by dilation/contraction of the medium arising from finite compressibility of the material. In the analytical solution the $\delta g_{3}$ is about double than in the numerical model $A$, where rocks maybe do not contract as much as in the half-space domain because of the less constraining steep topography. The total gravity change (Figs. $4 \mathrm{~d}$ and $5 \mathrm{~d}$ ) reaches a maximum amplitude of about 70 and $60 \mu$ Gal for both the analytical and the numerical model $A$, respectively. Discrepancies between analytical and numerical solutions are negligible as regards the field intensity, while notable changes in the pattern are observed. The gravity change, indeed, is wider and is strongly modified mainly in the summit area and in the eastern flank of Etna. The gravity field extends about 3-4 km from the magma intrusion and does not show significant changes at the gravity benchmarks, where in agreement with the models no gravity variations were recorded.

The values of chi-square for deformation components and magnetic changes are calculated to quantify the fit with the observed geophysical changes (Table I). The estimated measurement errors are $0.2 \mathrm{nT}$ for the magnetic changes and $0.005 \mathrm{~m}$ and $0.05 \mathrm{~m}$ for the horizontal and vertical deformation, respectively (Napoli et al., 2008; Aloisi et al., 2009). To enhance the match between the data and the numerical solutions including topography and medium heterogeneity, we changed some source parameters. In particular, we reduced the width of the dike until numerical solutions 
better fit the observed geophysical changes. In Fig. 6 the calculated deformation, magnetic and gravity fields are reported for the intrusive source with a width of $1 \mathrm{~km}$ (model B). The match between the observed and the computed magnetic changes is improved at most stations (Fig. 3). In particular, the computed piezomagnetic fields at BVD and PDN decrease in amplitude fitting better the magnetic observations. No significant differences are obtained at BCN. Also the ground deformation is slightly changed, even if the fit to the observed deformation is still acceptable (Table I). Indeed, magnetic field seems to be more sensitive to the change in source width.

Although these dislocation models are able to constrain the position and the geometry of the source, a constant-displacement discontinuity, in general, is not the most realistic description of a magma intrusion. An extension fracture is expected to result from magma overpressure on the dike surface and its opening is indeed found as part of the solution (Bonafede and Rivalta, 1999). Therefore, we performed new simulations where the source intrusion was represented by a mode I crack driven by a magmatic overpressure prescribed by constant-pressure boundary conditions (Gudmundson, 2008). We used the source geometry as in the numerical model B. We examine the values of the driving pressure, which fits the observed piezomagnetic changes and ground deformation. A preliminary estimate of the driving pressure $\Delta \mathrm{P}$ can be given by (Pollard and Segall, 1987):

$$
\Delta P=\frac{2 E<\Delta u_{I}>}{\pi W(1-v)^{2}}
$$

where $\left\langle\Delta u_{p}>\right.$ is the average opening of a $2 \mathrm{D}$ crack having a width $\mathrm{W}$ embedded in a homogeneous medium with a Young modulus $\mathrm{E}$. This 2D approximation does not account for free surface effects, and for the finite along strike dimension of the dike (Segall et al., 2001). In the crack model the estimate of the pressure change from ground deformation is directly related to the Young modulus. A decrease of the Young modulus requires a decrease of the driving pressure to achieve comparable deformation. On the contrary, when the pressure at the source wall is assigned, the piezomagnetic changes are not affected by variations in the rigidity of the host rocks but only in the initial magnetization (Currenti et al., 2009). Therefore, they can directly provide an estimate about the pressure change, if values of initial magnetization are known. Better resolution of the driving pressure is, therefore, related to the definition and accuracy of the magnetic and elastic medium parameters. Since the former are poorly known, it is advisable to consider values of the medium properties within reasonable ranges. Magnetic properties of volcanic rocks cropping out in the Etna volcano have rarely been measured; however, the few laboratory measurements carried out on recent and historic lava flows show values of remanent magnetization up to $9 \mathrm{~A} / \mathrm{m}$ (Pozzi, 1977; Tanguy, 1975; Del Negro and Napoli, 2002). The numerical simulations of the piezomagnetic field reveal that, for an initial magnetization ranging between 7 and $9 \mathrm{~A} / \mathrm{m}$ in the first layer, the related driving pressure varies from 15 to $12 \mathrm{MPa}$ in order to fit the observed magnetic changes (Table I). A lower overpressure would have required unreasonably high values of initial magnetization to 
obtain comparable piezomagnetic changes. Moreover, the range of overpressure values obtained from piezomagnetic evidence cannot provide the large observed deformations. Assuming an average magnetization of $8 \mathrm{~A} / \mathrm{m}$ and a related overpressure of $13 \mathrm{MPa}$ (model $\mathrm{C}$ ), the match with the magnetic observation is improved (Fig. 3), whereas the fit with ground deformation worsens (Table I). Indeed, the dike surface reaches a maximum opening of about $1.4 \mathrm{~m}$, which is lower than the $2 \mathrm{~m}$ expected from the dislocation model B. Therefore, a softer medium around the intrusion should be considered to enhance the deformation field. A better match is provided using a Young modulus of $10 \mathrm{GPa}$ (model D), which leads to a maximum opening at the center of the dike of about $2.4 \mathrm{~m}$ (Fig. 7). The assumption of a softer medium surrounding the intrusion in model D enhances the deformation with respect to model $C$, while the overpressure condition within the source provides approximately the same stress, thus maintaining the observed magnetic changes within the range of model $\mathrm{C}$ (Table I). The normal displacement along the dike surface is not uniform and shows an oval shape, as expected for a crack model (Fig. 7). The opening is greater on the east side of the dike with respect to the west, probably due to the mass deficit in the East sector in correspondence of the Valle del Bove depression.

\section{Discussion and Conclusion}

An integrated 3D model based on Finite Element Method (FEM) was developed and applied to review the 2008 magma intrusion detected at Mt Etna by magnetic data. The intrusive source obtained using the analytical model from magnetic data inversion by Napoli et al. (2008) was firstly used to guide parameter choices for the numerical model. Discrepancies between the analytical and the numerical model A are observed at the GPS stations located on the flanks of the volcano, since the intrusion is rather shallow and the altitude of the stations is lower than the intrusion top. Topography also significantly alters the general pattern of both piezomagnetic and gravity changes especially in proximity of steep cliffs of the summit area and of the eastern flank of Etna where the volcano edifice is rather asymmetric because of the depression of the Valle del Bove. The differences between the numerical and analytical gravity changes are less than $10 \mu \mathrm{Gal}$, which are below the measurements accuracy. Although changes in the gravity field are generally interpreted only in terms of additional mass input, our numerical results show that for a shallow magmatic intrusion the main contribution arises from the displacement of density boundaries. Moreover, the gravity changes highlight a butterfly pattern that is strongly different from the expected concentric pattern coming from the contribution of a mass input alone. Higher discrepancies between the numerical and analytical solutions are obtained in the intensity of the piezomagnetic field because of the stress concentration near rugged topography. While the topography is known with higher resolution and accuracy, the simple layered model is an over-simplification of the variation of the 
magneto-elastic parameters in the Etna subsurface. This assumption is due to the poor knowledge of magneto-elastic properties of Etna volcano. Moreover, the solutions could be strongly altered in presence of a more complex heterogeneity as revealed by comparison of different numerical models carried out by Currenti et al. (2009).

The source size was modified in the numerical model $B$ to obtain a better match between the observed and the computed geophysical variations at most stations. The numerical solution suggested an intrusive source with a reduced width in agreement with the shallow depth of departure of magma intrusion from the central conduit deduced by Aloisi et al. (2009).

The 2008 intrusion toward the northern flank was arrested in the summit area without downslope propagation and lava emission occurred only along the E-W eruptive fissure in the upper side of the Valle del Bove. Magma arrest depends strongly on the local stress in the different layers constituting the volcano edifice. The local stress is primarily determined by the loading conditions (tectonic stress, magmatic pressure, or displacement) and the elastic properties of the layers. Local discontinuities characterizing the rift zone may cause the arrest of magma at shallow crustal depths (Maccaferri et al., 2010). It is worth noting that magma intrusion propagates at a shallow depth, where the presence of very fractured and compliant rocks could have favored magma propagation through the medium. Considering that the overpressure of magma with respect to the horizontal stress in the host rock can play a key role for dike opening and propagation (Bonafede and Rivalta, 1999), it is essential to estimate the value or the range of values of driving pressure responsible for the intrusive process. In our numerical model the intrusion process was described in terms of pressure change, and the magma intrusion was modeled as a tensile crack. When the source is assigned in terms of the internal overpressure, the stress changes in the surrounding medium are better constrained than employing a dislocation source. Since the piezomagnetic effect depends directly on the deviatoric stress, it provides direct constraints on the internal overpressure of the intrusion. In this case, the piezomagnetic changes are slightly influenced by the elastic heterogeneity (Currenti et al., 2009) and they are nothing but a kind of stress measurement. Taking advantage of the observed piezomagnetic changes and assuming an initial magnetization of $8 \mathrm{~A} / \mathrm{m}$, which is an average reasonable value for Etnean rocks of the first layer where magma intruded, a driving pressure of $13 \mathrm{MPa}$ and a Young modulus of $10 \mathrm{GPa}$ were estimated (model D). The elastic model seems unable to reproduce the observed deformation, unless lower effective rigidity modulus ( $60 \%$ of the Young modulus assumed for the top layer) is considered. The effective rigidity concept is physically motivated by the hot, highly fractured and deformed shallow rocks surrounding the intrusion. The assumed elastic rheology may not be appropriated in proximity of the source where the medium behavior is better described using anelastic rheologies, such as viscoelastic and elastoplastic (Davis et al., 1979; Currenti et al., 2010; Jellinek et al., 2004). It is reasonable to expect that effectively viscous deformation of wall rocks, heated to well above the normal geotherm in proximity of magmatic sources, will influence 
the dynamics governing the formation of dikes (Jellinek and DePaolo, 2003; Del Negro et al., 2009). Non-linear rock behavior is consistent with laboratory tests carried out on Etnean rock samples (Rocchi et al., 2004) that revealed an average tensile strength of about $10 \mathrm{MPa}$ and can justify the wide fracture field produced in the northern flank of Etna during the magma propagation. Temporal evolution of magnetic changes, that started simultaneously at all stations and showed a fast decrease in the variations rate just an hour after the beginning of intrusion process (Napoli et al., 2008), highlighted an abrupt decrease of the driving pressure. This was plausibly caused by the simultaneous magma withdrawal from near effusive activity occurring in the Valle del Bove, and it can be considered as the main reason of the magma arrest.

The numerical model $D$ also revealed a non-homogeneous distribution of the displacement produced by intrusion. Particularly, the smallest displacement is observed along the upper west side of the dike where it is well confined by the volcano edifice, while to the east the mass deficit of the Valle del Bove better accommodated the stress field generated by magma intrusion.

On the basis of the results reported in Table I, model $D$ gets better agreement with respect to model $C$ but it is still worse than model $B$, which seems to provide the best overall fit. However, the integration of laboratory measurements of rock strength and magnetization with the numerical results indicates that better grounded physically models, such as models $C$ and $D$, are to be explored in the future to improve the data-fit and better constrain the source location and geometry. The application of 3D FEM model allowed for more accurate interpretations and inferences in modeling-based assessments of geophysical changes associated with volcanic activity. Since the numerical solution is influenced by the medium properties, a better knowledge of density and magnetization distribution inside the volcanic edifice is essential for improvement of integrated numerical models that may discriminate the main factors influencing the energetic levels involved in the opening and propagation of dikes. This information could provide a better knowledge of the dike intrusion processes and the rheological properties of rocks in the near field of emplaced dikes. That contributes to a more accurate evaluation of the hazard associated with the onset of Etna lateral eruptions, which are usually supplied by magma through fractures opened by magmatic overpressure.

\section{Acknowledgements}

This study was undertaken with financial support from the V3-LAVA and V4-FLANK projects (DPCINGV 2007-2009 contract) and was developed in the frame of the TecnoLab, the Laboratory for the Technological Advance in Volcano Geophysics organized by DIEES-UNICT and INGV-CT. We are grateful to the Editor M. Jellinek and the referees M. Bonafede, M. Johnston and Y. Sasai, who provided constructive comments for improving the manuscript. 


\section{References}

Aloisi, M., Bonaccorso, A., Cannavo`, F., Gambino, S., Mattia, M., Puglisi G., Boschi, E. (2009) A new dyke intrusion style for the Mount Etna May 2008 eruption modelled through continuous tilt and GPS data. Terra Nova, 00, 1-6, 2009.

Bonaccorso, A., and P.M., Davis, Modeling of ground deformation associated with recent lateral eruptions: mechanics of magma ascent and intermediate storage at Mt. Etna, in Mt. Etna: Volcano laboratory, ed. A Bonaccorso, S. Calvari, M. Cotelli, C. Del Negro and S. Falsaperla, American Geophysical Union Monograph, 293-306, 2004.

Bonafede, M., Mazzanti, M., (1998). Modelling Gravity Variations Consistent with Ground Deformation in the Campi Flegrei Caldera (Italy), J. Volcan. Geotherm. Res., 81, 137-157.

Bonafede, M., Rivalta, E. (1999), On tensile cracks close to and across the interface between two welded elastic half-spaces, Geophys. J. Int. (1999) 138, 410-434

Bonforte, A., Bonaccorso, A., Guglielmino, F., Palano, M., and Puglisi, G., (2008): Feeding system and magma storage beneath Mt. Etna as revealed by recent inflation/deflation cycles, J. Geophys. Res., 113, B05406, doi:10.1029/2007JB005334.

Budetta, G., et al. (2008), Osservazioni gravimetriche e magnetiche. Aggiornamento dello stato di attivita` dell'Etna:15 maggio 2008, INGV Rep. UFGM2008/05, Ist. Naz. di Geofis. e Vulcanol., Rome

Cai, Y. and Wang, C.Y. (2005): Fast finite-element calculation of gravity anomaly in complex geological regions, Geophys. J. Int., 162, 696-708

Carbone, D., Currenti, G. and Del Negro, C. (2007): Elastic model for the gravity and elevation changes prior to the 2001 eruption of Etna volcano, Bull. Volcanol., 69, 553-562, DOI 10.1007/s00445-006-0090-5.

Charco, M., Camacho, A. G., Tiampo, K.F. and Fernandez, J. (2009) Spatiotemporal gravity changes on volcanoes: Assessing the importance of topography, Geophys. Res. Lett., 36, L08306, doi:10.1029/2009GL037160.

Chiarabba, C., Amato, A., Boschi, E., and Barberi, F., (2000): Recent seismicity and tomographic modeling of the Mount Etna plumbing system, J. Geophys. Res., 105(B5), 10,923-10,938.

Corsaro, R.A. and M. Pompilio (2004), Buoyancy-controlled eruption of magmas at Mt Etna, Terra Nova 16, 16-22.

Currenti, G., C. Del Negro, and G. Ganci (2007), Modelling of ground deformation and gravity fields using finite element method: An application to Etna volcano, Geophys. J. Int., 169, 775-786, doi:10.1111/j.1365-246X.2007.03380.x.

Currenti, G., Del Negro C., Di Stefano, A. and Napoli, R. (2009): Numerical simulation of stress induced piezomagnetic fields at Etna volcano, Geophys. J. Int., doi: 10.1111/j.1365-246X.2009.04381.x

Currenti, G., Bonaccorso, A., Del Negro, C. and Scandura, D. (2010), Elasto-plastic modeling of volcano ground deformation, with application on Mt Etna, Earth Planet. Sci. Lett., 296, 311-318, doi:10.1016/j.epsl.2010.05.013

Del Negro, C., and R. Napoli (2002), Ground and marine magnetic surveys of the lower eastern flank of Etna volcano (Italy), J. Volcanol. Geotherm. Res., 114(3-4), 357- 372. 
Del Negro, C., and G. Currenti (2003), Volcanomagnetic signals associated with the 2001 flank eruption of Mt. Etna (Italy), Geophys. Res. Lett., 30(7), 1357, doi:10.1029/2002GL015481.

Del Negro, C., G. Currenti, R. Napoli, and A. Vicari (2004), Volcanomagnetic changes accompanying the onset of the 2002- 2003 eruption of Mt. Etna (Italy), Earth Planet. Sci. Lett., 229, 1 - 14.

Del Negro, C., G. Currenti, and D. Scandura (2009), Temperature-dependent viscoelastic modeling of ground deformation: application to Etna volcano during the 1993-1997 inflation period. Phys. Earth Planet. In., 172, 3-4, 299-309.

Di Grazia, G., Cannata, A., Montalto, P., Patane`, D., Privitera, E., Zuccarello, L., Boschi, E. (2009), A multiparameter approach to volcano monitoring based on $4 \mathrm{D}$ analyses of seismo-volcanic and acoustic signals: The 2008 Mt. Etna eruption, Geophys. Res. Lett, 36, L18307, doi:10.1029/2009GL039567, 2009.

Ebinger,C., Ayele, A., Keir, D., Rowland, J., Yirgu, G., Wright, T., Belachew, M., and Hamling, I. (2010) Length and Timescales of Rift Faulting and Magma Intrusion: The Afar Rifting Cycle from 2005 to Present, Annu. Rev. Earth Planet. Sci. 38:439-66

Furuya, M., Okubo, S., Sun, W., Tanaka, Y., Oikawa, J., Watanabe, H., Maekawa, T. (2003) Spatiotemporal gravity changes at Miyakejima Volcano, Japan: Caldera collapse, explosive eruptions and magma movement, J. Geophys. Res., 108(B4), doi:10.1029/2002JB001989.

Greco, F., C. Carmisciano, C. Del Negro, I. Loretti, A. Sicali, and P. Stefanelli (2008), Seismic-induced accelerations detected by two coupled gravity meters in continuous recording with a high sampling rate at Etna volcano, Annals of Geophysics, 51, 1.

Gudmundsson, A., Friese, N., Galindo, I., Philipp, S. L. (2008), Dike-induced reverse faulting in a graben, Geology, 2008, 36, 123-126, doi: 10.1130/G24185A.1.

Hagiwara, Y., 1977. The Mogi model as a possible cause of the crustal uplift in the eastern part of Izu Peninsula and related gravity change, Bull. Earthq. Res. Inst., Univ. Tokyo, 52, 301-309.

Hinderer, J., and D. Crossley (2000), Time variations and inferences on the Earth's structure and dynamics, Surv. Geophys. 21, 1-45.

Jellinek, A.M., M. Manga, M.O. Saar (2004), Did melting glaciers cause volcanic eruptions in eastern California? Probing the mechanics of dike formation, J. Geophys. Res., 109, B9, B09206, doi: 10.1029/2004JB002978,.

Jellinek, A.M. and D.J DePaolo (2003), A model for the origin of large silicic magma chambers: Precursors of catastrophic caldera-forming eruptions, Bull. Volcanol. 65, 363-381.

Maccaferri, F., Bonafede, M. and Rivalta, E., (2010) A numerical model of dyke propagation in layered elastic media, Geophys. J. Int. (2010) 180, 1107-1123, doi: 10.1111/j.1365-246X.2009.04495.x

Nagata, T., 1970. Basic magnetic properties of rocks under mechanical stresses, Tectonophysics, 9, 167195.

Napoli, R., Currenti, G., Del Negro, C., Greco, F. and Scandura , D., (2008): Volcanomagnetic evidence of the magmatic intrusion on 13th May 2008 Etna eruption, Geophys. Res. Lett.,35, L22301, doi:10.1029/2008GL035350.

Okubo, S., 1992. Gravity and Potential Changes due to Shear and Tensile Faults in a Half-Space, J. Geoph. Res., 97(B5), 7137-7144. 
Okubo, A. and Oshiman, N., 2004. Piezomagnetic field associated with a numerical solution of the Mogi model in a non-uniform elastic medium. Geophys. J. Int., 159, 509-520.

Pollard, D.D., and P. Segall, Theoretical displacements and stresses near fractures in rock: with application to faults, joints, veins, dikes, and solution surfaces, In Fracture Mechanics of Rock, Academic Press, $p$. 227-349, 1987.

Pozzi, J.P. 1977. Effects of stresses on magnetic properties of volcanic rocks, Phys. Earth planet Inter., 14, 77-85.

Rocchi, V., Sammonds, P.R., Kilburn, C.R.J. (2004), Fracturing of Etnean and Vesuvian rocks at high temperatures and low pressures, J. Volc. Geother. Res. 132, 137-157

Sasai, Y., 1986. A Green's function for tectonomagnetic problems in an elastic half-space, J. Geomag. Geoelectr., 38, 949-969.

Sasai, Y., (1991), Tectonomagnetic modeling on the basic of the linear piezomagnetic effect, Bull. Earthq. Res. Inst., Univ. Tokyo, 66, 585-722.

Schiavone, D. and M. Loddo (2007), 3-D density model of Mt. Etna Volcano (Southern Italy), J. Volcanol. Geoth. Res. 164, 161-175.

Segall, P., Cervelli, P., Owen, S., Lisowski, M., Miklius, A., (2001) Constraints on Dike Propagation from Continuous GPS Measurements, Geophys. Res., 106, 19,301-19,318.

Tanguy, J.C. 1975. Intensity of the geomagnetic field from recent Italian lavas using a new paleointensity method. Earth planet Sci. Lett., 27, 314-320.

Tibaldi, A. and Groppelli, G., (2002), Volcano-tectonic activity along structures of the unstable ne flank of Mt. Etna (Italy) and their possible origin, J. Volcan. Geotherm. Res., 115, 277-302.

Utsugi, M., Nishida, Y., and Sasai, Y., (2000), Piezomagnetic potentials due to an inclined rectangular fault in a semi-infinite medium, Geophys. J. Int., 140, 479-492. 
Table

\begin{tabular}{lccccc}
\hline & Analytic Model & Model A & Model B & Model C & Model D \\
\hline Magnetic & 255 & 766 & 150 & 140 & 140 \\
Horizontal deformation & 426 & 396 & 405 & 1650 & 557 \\
Vertical deformation & 609 & 487 & 350 & 1200 & 423 \\
\hline
\end{tabular}

Table I - Chi-square values of deformation and magnetic changes for the analytical and the numerical models. 
Figure 1 - Schematic map of the Etna summit area covered by the lava flows of the 2008 eruption. Locations of magnetic, gravity and GPS stations are also shown. Inset shows the position of the CSR magnetic reference station.

Figure 2 - Comparison between analytical solutions (a) and numerical results of model A (b) for the 2008 intrusive source from Napoli et al. [2008]. Piezomagnetic change (contour lines at 2 nT) generated by the intrusive dike (black line). Observed (blue arrows) and computed (red arrows) deformation at the permanent GPS stations are also reported. The recorded magnetic changes are reported in the inset.

Figure 3 - Comparison between measured and computed magnetic changes for the analytical and numerical models.

Figure 4 - Gravity contributions generated by the intrusive source using the analytical solution. Contour intervals are $1 \mu \mathrm{Gal}$ for $\delta g_{1}(\mathrm{a}), 10 \mu \mathrm{Gal}$ for $\delta g_{2}$ (b), $2 \mu \mathrm{Gal}$ for $\delta g_{3}$ (c) and $10 \mu \mathrm{Gal}$ for the total gravity change (d). Black circles represent the gravity stations of the monitoring network at Mt. Etna.

Figure 5 - Gravity contributions generated by the intrusive source using the numerical model A. Contour intervals are $1 \mu \mathrm{Gal}$ for $\delta g_{1}(\mathrm{a}), 10 \mu \mathrm{Gal}$ for $\delta g_{2}$ (b), $2 \mu \mathrm{Gal}$ for $\delta g_{3}$ (c) and $10 \mu \mathrm{Gal}$ for the total gravity change (d). Black circles represent the stations of the gravity monitoring network at Mt. Etna.

Figure 6 - Piezomagnetic changes, ground deformation (a), and gravity changes (b) caused by the magmatic intrusion in model B. Observed (blue arrows) and computed (red arrows) deformation at the permanent GPS stations are also reported. Contour intervals are $2 \mathrm{nT}$ for the piezomagnetic changes (a) and $10 \mu \mathrm{Gal}$ for gravity changes (b).

Figure 7 - Normal displacement to the dike wall in the numerical model D. The dilation profile (opening exaggerated by a factor 300 ) for an overpressure of $13 \mathrm{MPa}$ is also reported (black line). 


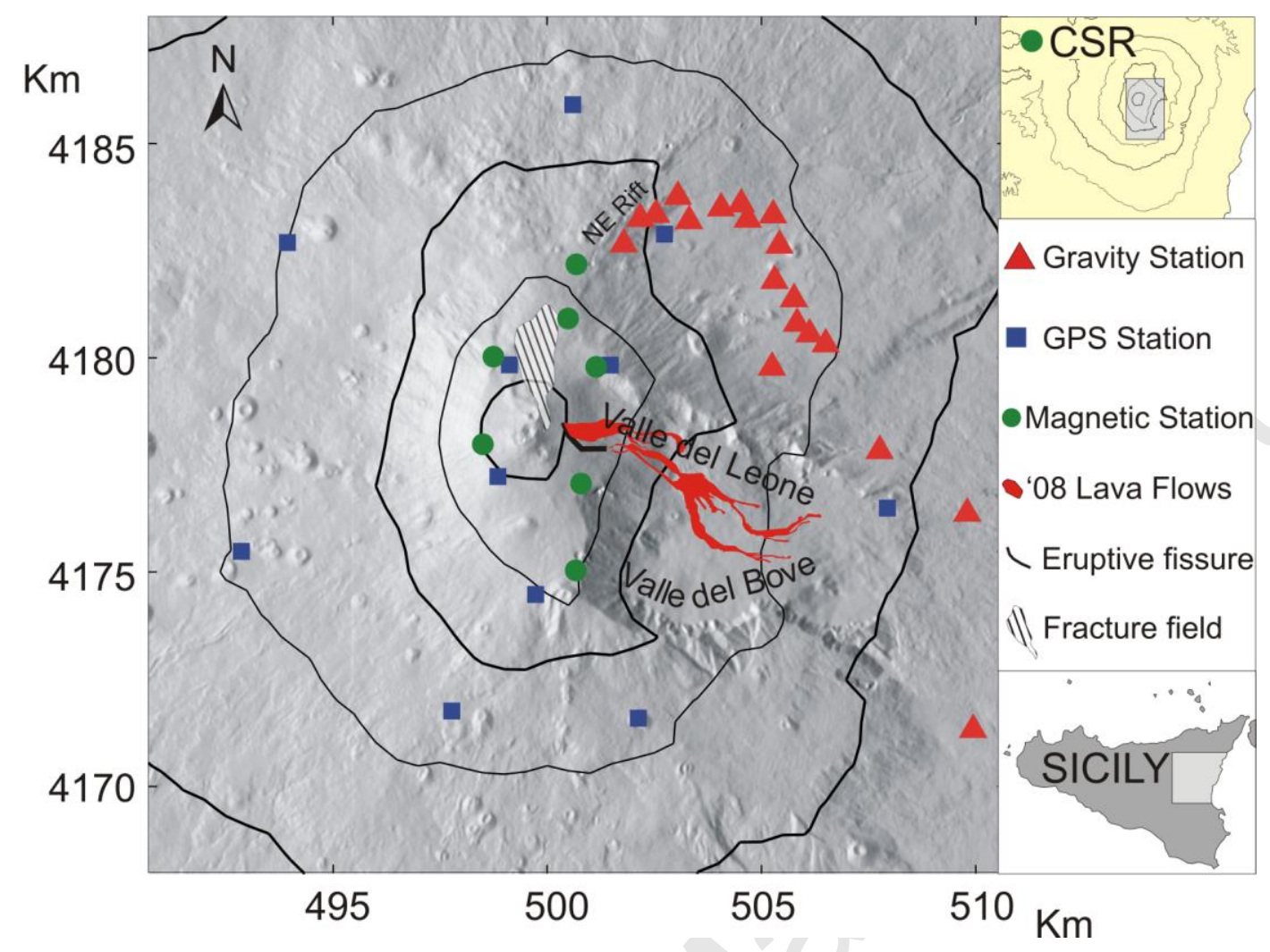

Figure 1 - Schematic map of the Etna summit area covered by the lava flows of the 2008 eruption. Locations of magnetic, gravity and GPS stations are also shown. Inset shows the position of the CSR magnetic reference station. 


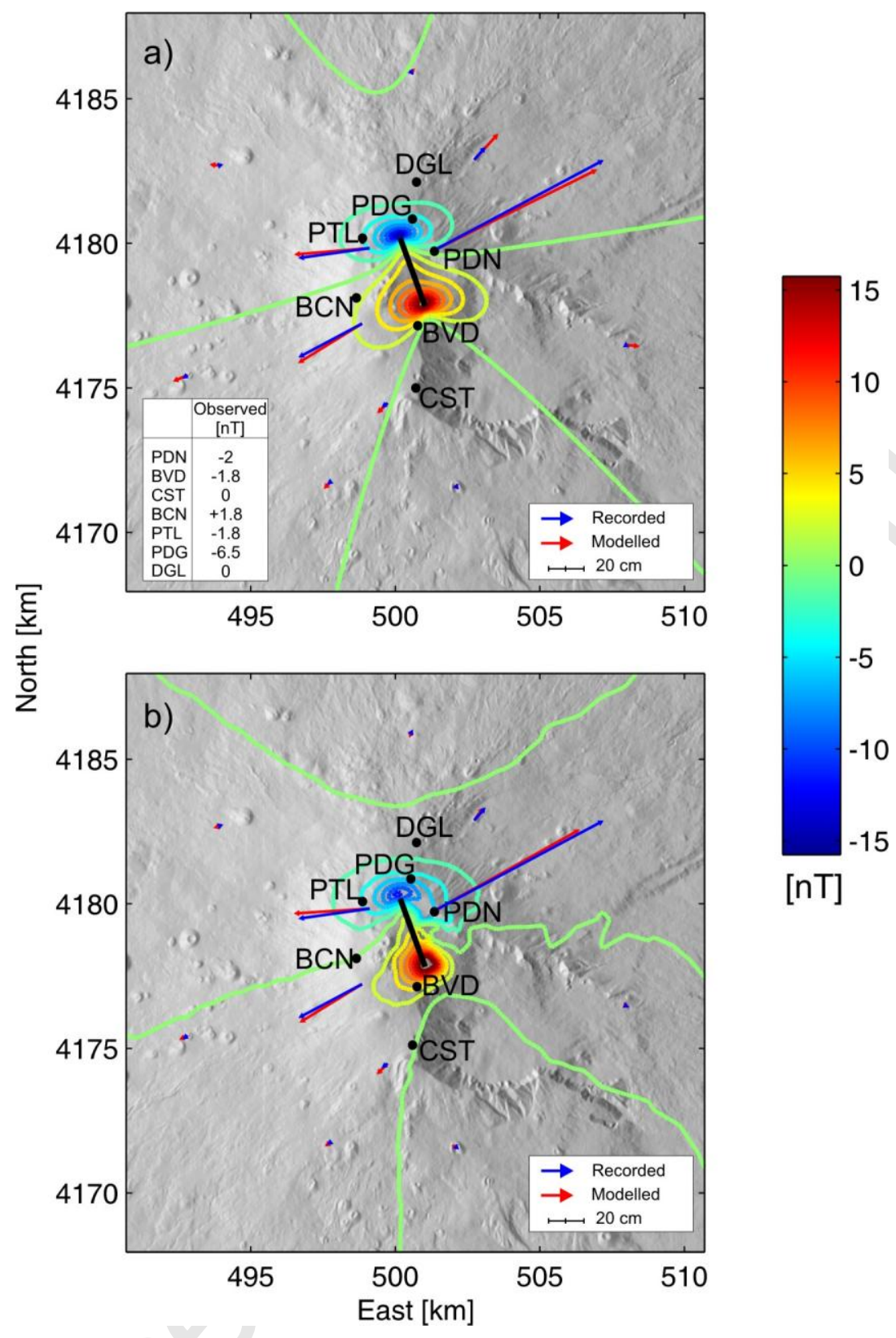

Figure 2 - Comparison between analytical solutions (a) and numerical results of model $A(b)$ for the 2008 intrusive source from Napoli et al. [2008]. Piezomagnetic change (contour lines at 2 nT) generated by the intrusive dike (black line). Observed (blue arrows) and computed (red arrows) deformation at the permanent GPS stations are also reported. The recorded magnetic changes are reported in the inset. 


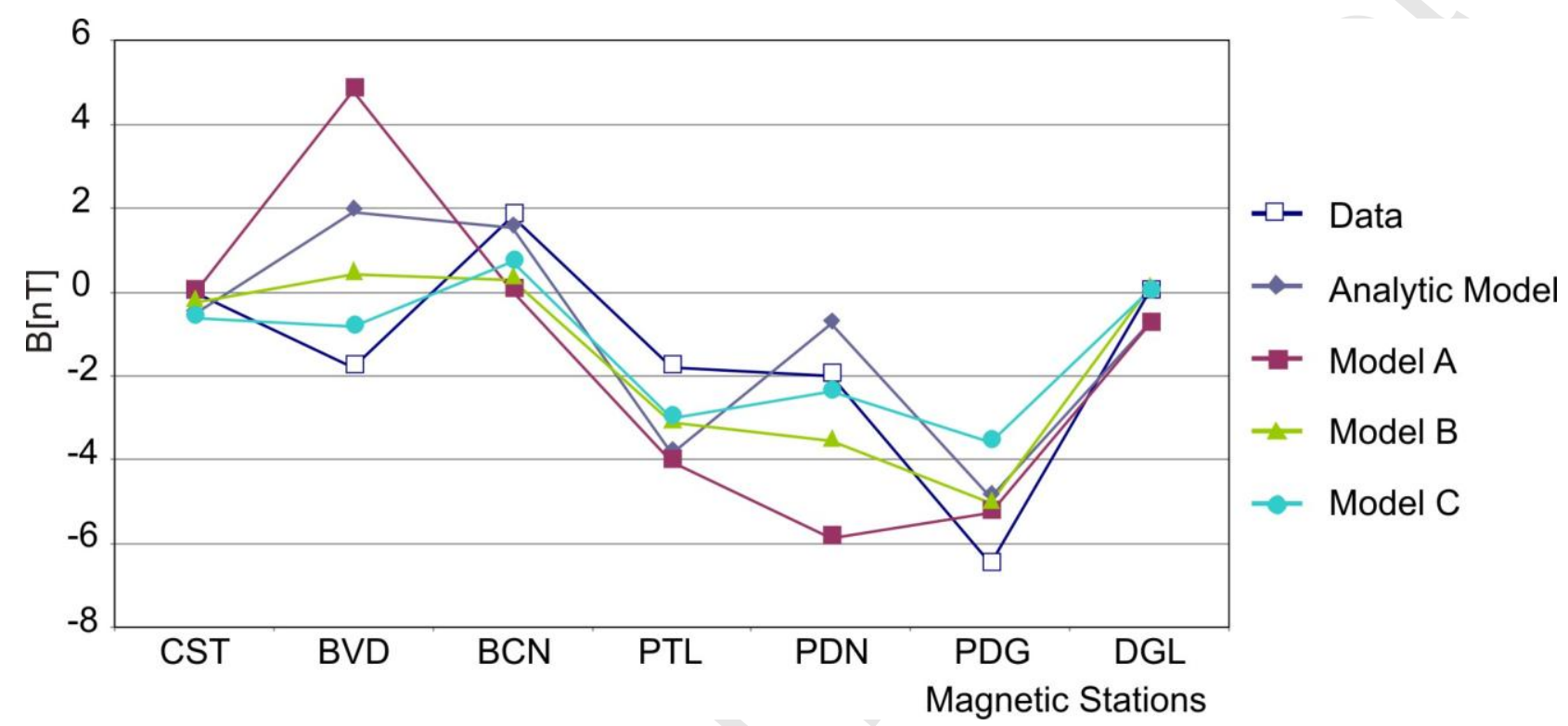

Figure 3 - Comparison between measured and computed magnetic changes for the analytical and numerical models. 


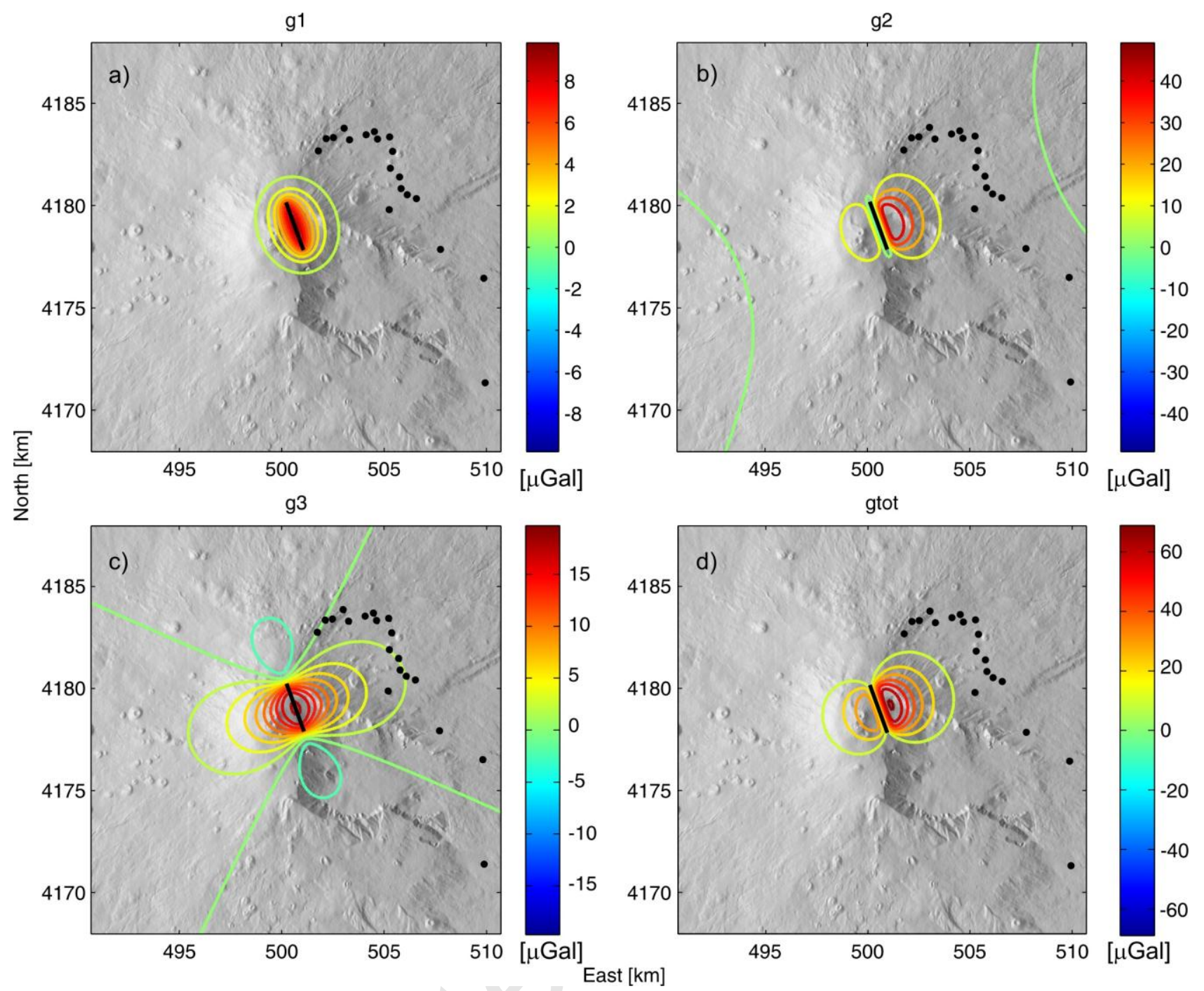

Figure 4 - Gravity contributions generated by the intrusive source using the analytical solution. Contour intervals are $1 \mu \mathrm{Gal}$ for $\delta g_{1}$ (a), $10 \mu \mathrm{Gal}$ for $\delta g_{2}$ (b), $2 \mu \mathrm{Gal}$ for $\delta g_{3}$ (c) and $10 \mu \mathrm{Gal}$ for the total gravity change (d). Black circles represent the gravity stations of the monitoring network at Mt. Etna. 


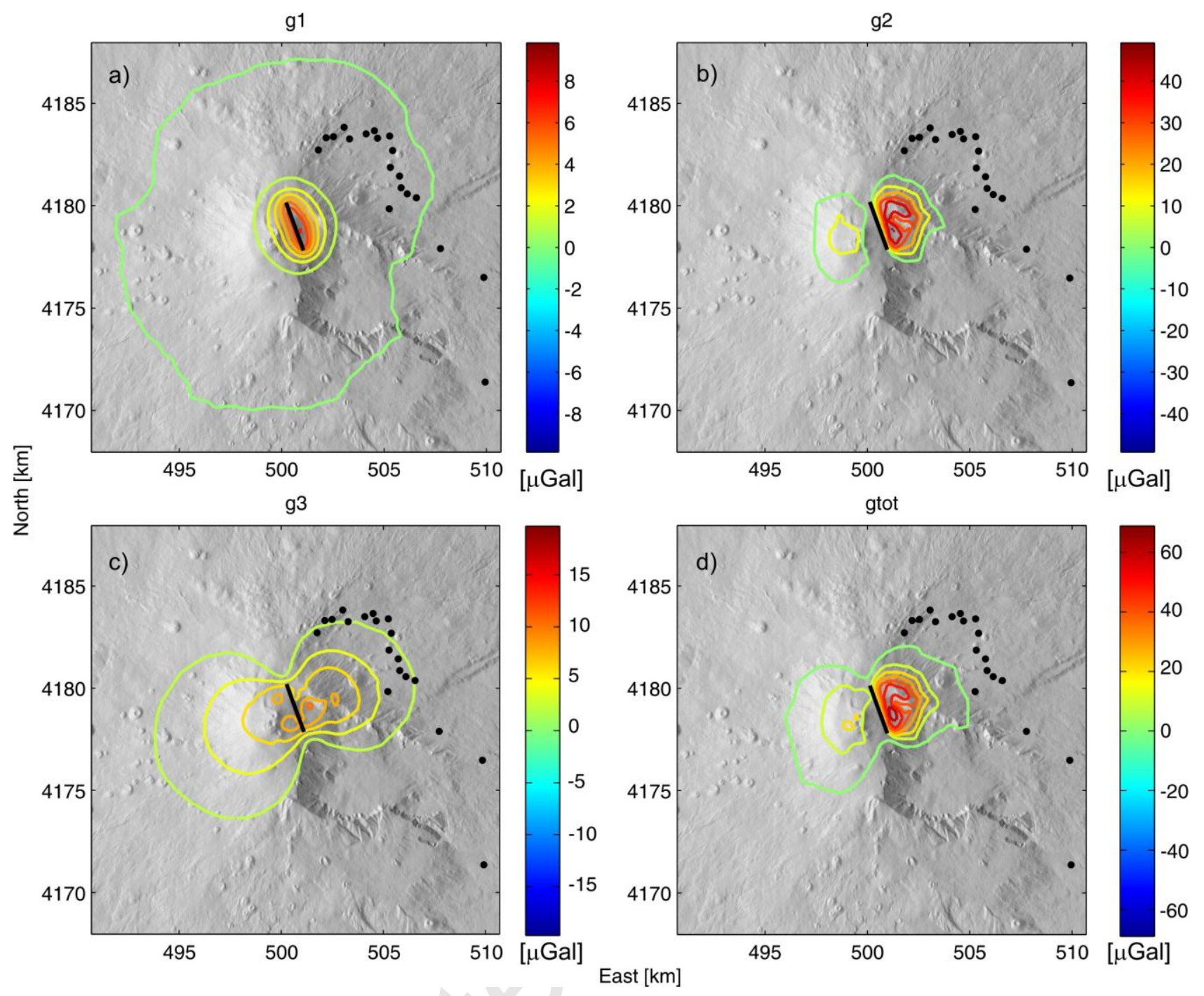

Figure 5 - Gravity contributions generated by the intrusive source using the numerical model A. Contour intervals are $1 \mu \mathrm{Gal}$ for $\delta g_{1}$ (a), $10 \mu \mathrm{Gal}$ for $\delta g_{2}$ (b), $2 \mu \mathrm{Gal}$ for $\delta g_{3}$ (c) and $10 \mu \mathrm{Gal}$ for the total gravity change (d). Black circles represent the stations of the gravity monitoring network at Mt. Etna. 


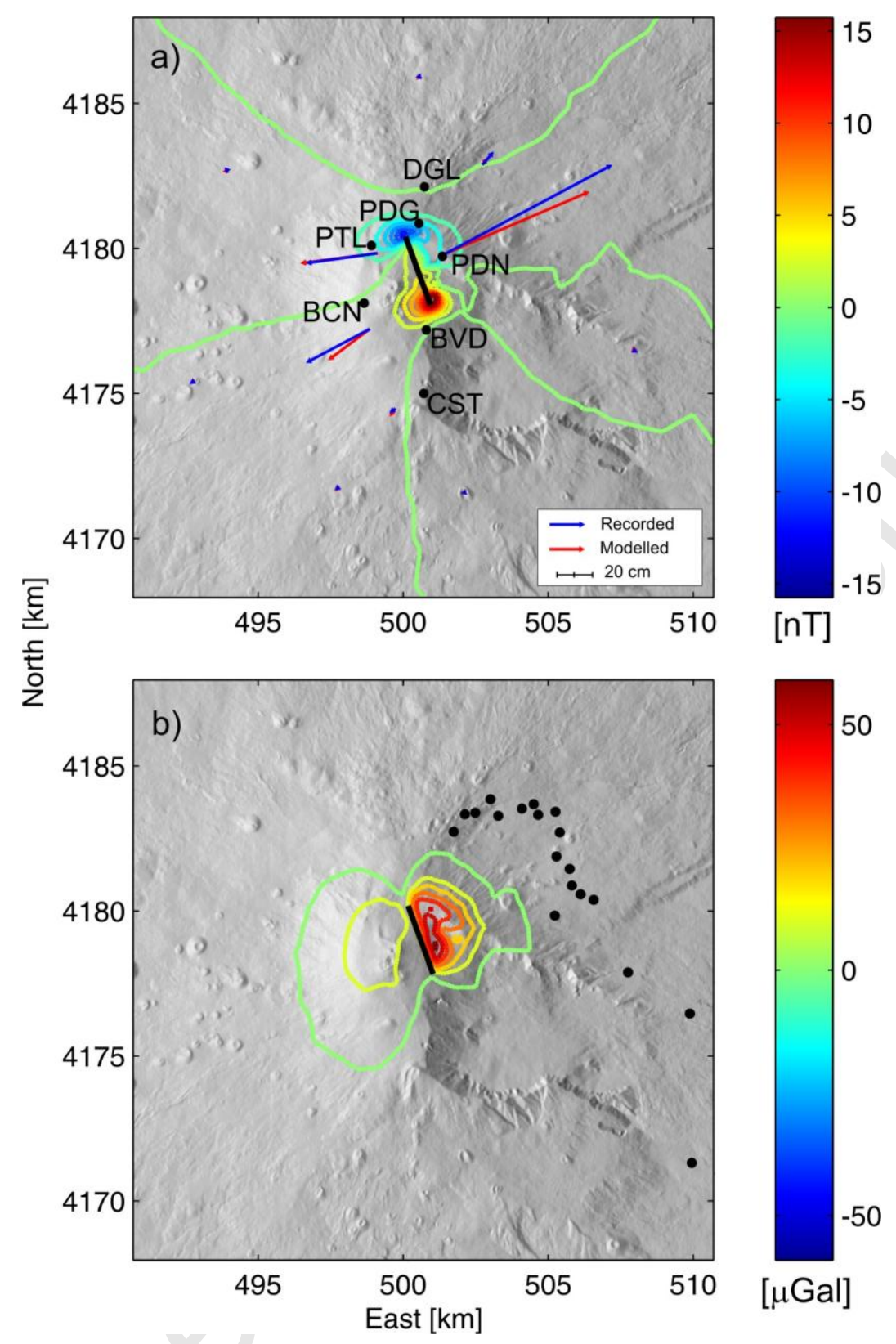

Figure 6 - Piezomagnetic changes, ground deformation (a), and gravity changes (b) caused by the magmatic intrusion in model B. Observed (blue arrows) and computed (red arrows) deformation at the permanent GPS stations are also reported. Contour intervals are $2 \mathrm{nT}$ for the piezomagnetic changes (a) and $10 \mu \mathrm{Gal}$ for gravity changes (b). 


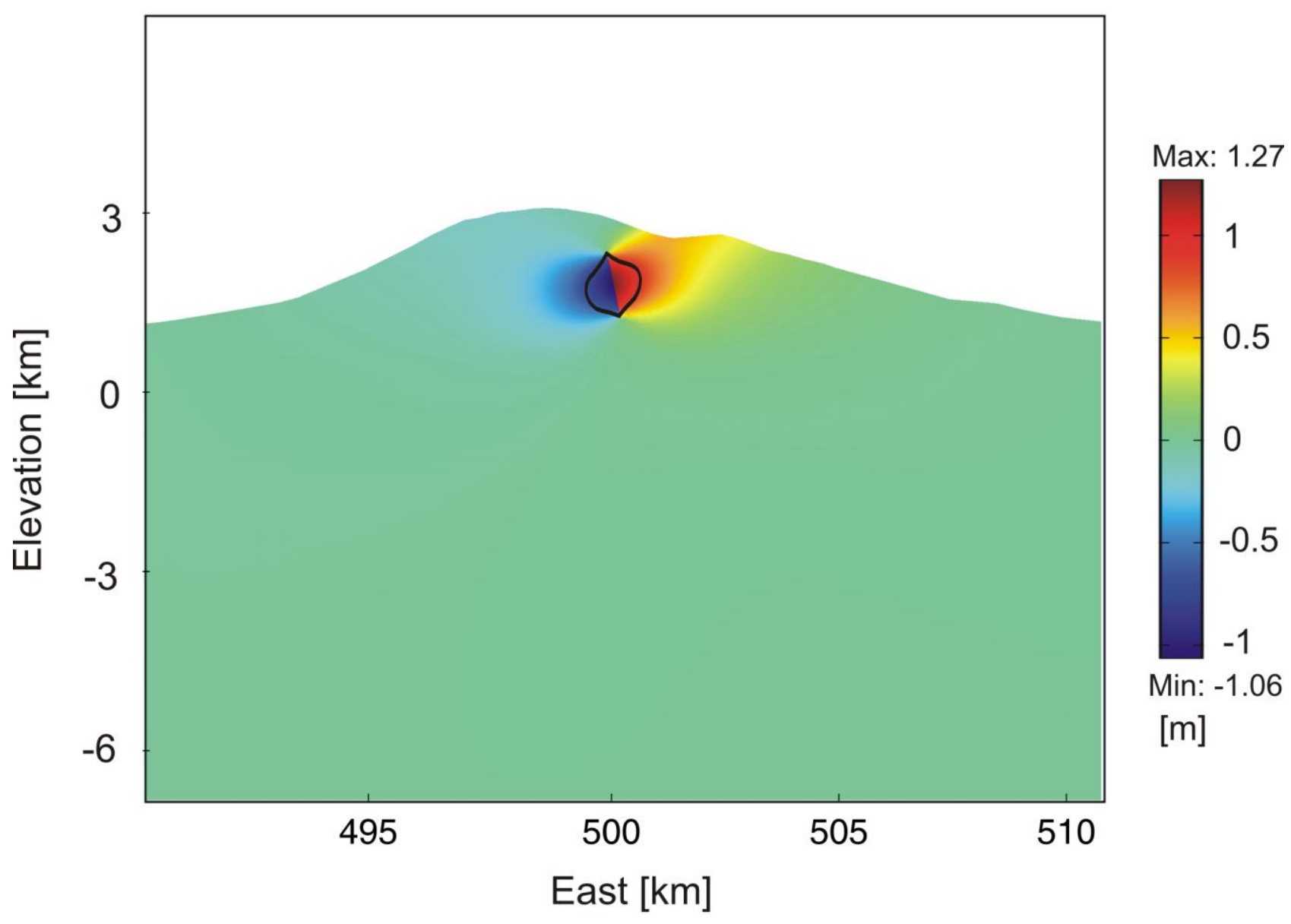

Figure 7 - Normal displacement to the dike wall in the numerical model D. The dilation profile (opening exaggerated by a factor 300 ) for an overpressure of $13 \mathrm{MPa}$ is also reported (black line). 